\title{
Sericin Peptide Attenuates Non-Alcoholic Steatohepatitis Via Mitochondrial Function Modulation
}

\section{Na Ryung Choi ${ }^{1}$, Hyeon Tae Kang ${ }^{2}$, Seung Min Lee², Yeon-Lim Suh ${ }^{3}$, Hyukjin Kwon ${ }^{4}$, Jaekyu Noh ${ }^{4}$, Sang Bong Ahn ${ }^{5}$ and Dae Won Jun ${ }^{1,2 *}$}

${ }^{1}$ Department of Internal Medicine, Hanyang University School of Medicine, Seoul, Republic of Korea

${ }^{2}$ Department of Translational Medicine Hanyang University Graduate School of

Biomedical Science and Engineering, Seoul, Republic of Korea

${ }^{3}$ Department of Pathology, Samsung Medical Center, Sungkyunkwan University

School of Medicine, Seoul, Republic of Korea

${ }^{4}$ NI\&Pharm INC, Seoul, Republic of Korea

${ }^{5}$ Department of Internal Medicine, Eulji University School of Medicine, Seoul,

Republic of Korea

*Corresponding Author: Dae Won Jun, Department of Internal Medicine,

Hanyang University College of Medicine, Republic of Korea.
Received: July 20, 2020

Published: August 24, 2020

(C) All rights are reserved by Dae Won Jun., et al.

\begin{abstract}
Sericin is a component of cocoons. Studies have shown that sericin improves metabolic disease in animal model. However, it is not yet known whether sericin is effective in the treatment of non-alcoholic steatohepatitis. Twenty C57BL/6N mice were divided into control and sericin treated group. High fat diet was fed to mice for 10 weeks. From $11^{\text {th }}$ to $21^{\text {st }}$ week sericin and distilled water were administered assigned groups via oral gavage. Body weight and liver to body weight ratio was assessed. AST, ALT, triglyceride, cholesterol and blood glucose levels were measured. qRT-PCR, Electro-microscopy, and Free fatty acid induced lipotoxicity assay were performed. Mitochondrial membrane potential was checked. As a result, there were no differences in body weight and diet intake in two groups. However, liver weight and liver/body weight ratio were significantly decreased in sericin group than in control group. Both the degree of hepatic steatosis and inflammation were lower in sericin group than the control group. NAFLD activity score were also decreased in treatment group. Serum ALT, AST, and triglyceride levels as well as area under receiver operating characteristics of oral glucose tolerance test were significantly decreased in sericin group. Hepatic mRNA expressions of TNF- $\alpha$, MCP-1, and IL-6 mRNA were decreased in sericin group. Electron microscopy findings showed restoration of abnormal mitochondria with sericin treatment. Lipotoxicity induced HepG2 cell death was significantly attenuated in palmitic acid and sericin co-treated cells. Sericin also prevented palmitic acid induced mitochondrial depolarization. In conclusion, Sericin peptide treatment attenuated non-alcoholic steatohepatitis via mitochondrial function modulation.
\end{abstract}

Keywords: Sericin; Nonalcoholic steatohepatitis; Mitochondria

\section{Abbreviations}

ALT: Alanine Transaminase; AST: Aspartate Aminotransferase; AUC: Area Under the Curve; FAS: Fatty Acid Synthase; IL-6: Inter- leukin 6; LDL: Low-density Lipoprotein; MCP-1: Monocyte Chemoattractant Protein-1; NAFLD: Non-Alcoholic Fatty Liver Disease; NAS: NAFLD Activity Score; NASH: Non-alcoholic Steatohepatitis; 
OGTT: Oral Glucose Tolerance Test; ROS: Reactive Oxygen Species; qRT-PCR: Quantitative Real Time Polymerase Chain Reaction; SCD1: Stearoyl-CoA desaturase-1; SREBP1c: Sterol-regulatory-element-binding protein-1c; TG: Triglyceride; TNF- $\alpha$ : Tumor Necrosis Factor Alpha; VLDL: Very-low-density Lipoprotein

\section{Introduction}

Non-alcoholic fatty liver disease (NAFLD) is a common disease in the Orient accounting for $29.6 \%$ of Asian prevalence and has been steadily increasing in recent years [1]. NAFLD is known to be caused by a multiplicity of factors. Among these, mitochondrial dysfunction is known to play an important role in the development of NAFLD [2,3]. Mitochondrial dysfunction contributes to NAFLD pathophysiology because it affects fat accumulation in liver and promotes reactive oxygen species (ROS) production, lipid peroxidation, cytokine release, and cell necrosis [4]. In addition, uncoupling of oxidative phosphorylation, production of free radicals, and lipid peroxidation cause cell damage. ROS production leads to lipid peroxidation in mitochondrial membranes, which impairs mitochondrial function. Oxidative stress also triggers inflammatory cytokine production, inflammation, and fibrosis. This ultimately results in progression toward non-alcoholic steatohepatitis (NASH) [5].

The silk protein, sericin, is a constituent of silkworm cocoons accounting for 20 to $30 \%$ of the total cocoon weight. It is made in the middle silk gland of silkworm Bombyx mori. Sericins encase fibroins that form the central structure of cocoon and make them stick together. Sericins are almost completely removed from cocoon during preparation of silk fabrics [6]. Previous studies have shown that sericin is beneficial to health because it has antioxidant, anticancer, and skin regeneration effects [7].

A study by Ampawong., et al. evaluated the effects of sericin on fatty liver and showed that sericin did not lower blood glucose levels but had hypocholesterolemic effects. In addition, sericin treatment resulted in a decreased number of dysmorphic mitochondria in the heart and liver of high-cholesterol diet/streptozotocin-treated rats [8]. The study also found that sericin-treated group had a reduced number of abnormal endoplasmic reticuli in the exocrine pancreas and exhibited preserved zymogen granules and cytoplasmic organization. The treatment also decreased microvesicular steatosis by reducing fat deposits caused by hypercholesterolemic stage in liver.
Although previous studies showed antioxidative properties of sericin, it is still not unclear whether sericin could attenuate hepatic inflammation, steatosis and hepatic fibrosis. Although few studies evaluated the in vivo mechanism of sericin action; however, to best of our knowledge, no study evaluated the effects of sericin treatment in a NASH model.

\section{Aim of the Study}

The aim of our study was to investigate the effect of sericin on NASH improvement using animal NASH model.

\section{Materials and Methods}

\section{Study design}

Twenty (6-week-old, C57BL/6N) mice were purchased from Orientbio (Seongnam, Republic of Korea); the animals were maintained under constant temperature $\left(23 \pm 2^{\circ} \mathrm{C}\right)$ and humidity $(55$ $\pm 5 \%$ ) conditions as well as $12: 12 \mathrm{~h}$ light/dark cycles. A high-fat diet (Rodent Diet with 60 kcal\% fat, D12492, Joong-ah Bio, Suwon, Republic of Korea) was fed to all 20 mice for 21 weeks. At the end of $10^{\text {th }}$ week, the mice were divided into two groups ( $\mathrm{n}=10$ each); control (NASH) and sericin (NASH+S) groups (Figure 1A). Distilled water and sericin $(1,600 \mathrm{mg} / \mathrm{kg})$ were administrated via oral gavage from $11^{\text {th }}$ to $21^{\text {st }}$ weeks. Body weight and food intake were measured weekly. All mice were sacrificed at the end of experiment. Animals were anesthetized by intraperitoneal injecting a 1: 1: 2 of Zoletil (Tiletamine+Zolazepam), Rompun (Xylazine) solution and were sacrificed by cervical dislocation. Liver tissue and blood samples were collected; liver weight and liver to body weight ratios were recorded. The liver tissue samples were stored in $4 \%$ formalin at $4^{\circ} \mathrm{C}$ until histological analysis or at $-80^{\circ} \mathrm{C}$ until molecular biological analysis.

\section{Oral glucose tolerance test (OGTT)}

The day before sacrifice, the animals were fasted 5 hours before the OGTT and their body weights were measured. In relation to body weight, $20 \%$ volume of dextrose was calculated for each mouse. The blood glucose levels of mice were measured from tailsnip blood using glucometer (Accu-Chek, Roche). After first glucose measurement, the dextrose was administrated by oral gavage to each mouse and glucose levels were measured after $30,60,90$, and $120 \mathrm{~min}$.

\section{Biochemical analyses}

Blood samples were collected into serum separation tubes (Vacutainer SST II Plastic serum tube, BD367955, Becton-Dickinson \& 
Co., San Jose, CA) and serum was separated by centrifugation at 3,000 rpm for $15 \mathrm{~min}$. serum were then transferred to new eppendorf tubes. Analyses of alanine transaminase (ALT), aspartate aminotransferase (AST), triglyceride (TG), albumin, and cholesterol levels were performed by an animal sample diagnosis company: KNOTUS Co. Ltd. (Guri, Republic of Korea).

\section{Histological analysis}

Liver tissues were fixed in $4 \%$ neutral formalin and were embedded in the paraffin and sliced into $4-\mu \mathrm{m}$ sections. Tissue sections were then stained with hematoxylin and eosin to evaluate NAFLD activity scores (NAS) and observed under a light microscope (Leica DM 4000B, Wetzlar, Germany).

Total NAS score represents the sum of the scores of steatosis, lobular inflammation, and ballooning, and the range is $0-8$. We first quantified the NASH according to NAS grading system for histological activity of liver tissue. The range of NAS scores considered to diagnostic for NASH is 5-8 points [9].

Amount steatosis surface area was evaluated on low power examination; minimal steatosis $(<5 \%)$ received a score of $0,5-33 \%$ steatosis received score $1,34-66 \%$ received score $2,>66 \%$ received score 3. Lobular inflammation was divided into following four category; no foci as evaluated on 200x magnification to score of 0,1 foci to score of $1,2-4$ foci to score of 2 , more than 4 foci to score of 3 . In hepatocyte ballooning, none is score of 0 , few balloon cells are count as score 1, many cells or prominent ballooning is count as score 2 .

Furthermore, Sirius Red staining was performed using PicroSirius Red Stain Kit (Abcam, Cambridge, UK) according to manufacturer's instructions to compare liver fibrosis in two groups. When stained, the collagen fibers were represented as red. The quantification of staining area was done with ImageJ program using five randomly selected fields per section (magnification $\times 100$ ). The scale of the image was changed to micrometers by ImageJ. The images were converted to grayscale; the threshold was set to half the automatically set threshold. The area and percentage area of red-stained collagen was measured using the set threshold. The values were represented as means \pm standard deviations.

Additionally, fibrosis was histologically quantified as follows: 0 , none; $1 \mathrm{a}$, perisinusoidal or portal/periportal, mild; $1 \mathrm{~b}$, perisinusoidal or portal/periportal, moderate; $1 \mathrm{c}, 2$ :perisinusoidal and portal/periportal 3, bridging fibrosis] [9]. We converted 1a to score of $1.25,1 \mathrm{~b}$ to score of $1.5,1 \mathrm{c}$ to score 1.75 and then performed parametric test with unpaired t-test.

Quantitative real-time PCR analysis

RNA extracted from the liver tissues was subjected to reverse transcription and subsequent PCR using LightCycler ${ }^{\circledR} 480$ system (Roche Diagnostics, Mannheim, Germany) with LightCycler ${ }^{\circledR} 480$ SYBR GreenIMaster mix (Roche Diagnostics). Primer sequences are listed in the table below. Target gene expression was normalized to GAPDH expression levels as an internal control.

\begin{tabular}{|c|c|c|}
\hline Gene & Forward & Reverse \\
\hline$T N F-\alpha$ & ACG GCA TGG ATC TCA AAG & CGG ACT CCG CAA AGT CTA \\
\hline$M C P-1$ & CCC AAT GAG TAG GCT GGA & TCT GGA CCC ATT CCT TCT \\
\hline$I L-6$ & CCG GAG AGG AGA CTT CAC & TCC ACG ATT TCC CAG AGA \\
\hline SREBP1 & GAA ACA CTC AGC AGC CAC & CAA GCT TTG GAC CTG GGT \\
\hline FAS & CCC TTT TTG AGG AGG CCA & GCT TCA CGA CTC CAT CAC \\
\hline$S C D-1$ & AGA AGG GCG GAA AAC TGG & AGG CCG GGC TTG TAG TAC \\
\hline$G A P D H$ & $\begin{array}{c}\text { GTT GTC TCC TGC GAC TTC } \\
\text { A }\end{array}$ & $\begin{array}{c}\text { GGT GGT CCA GGG TTT } \\
\text { CTT A }\end{array}$ \\
\hline
\end{tabular}

Table 1

\section{Electron microscopic studies}

Electron microscopic study was performed using formalin-fixed, paraffin embedded liver tissue. Paraffin-embedded liver samples were retrieved, rehydrated, post-fixed in osmium tetroxide, and embedded in Epon-Araldite (Ladd Research Industries, Williston, VT). Following this procedure, 1-mm-thick tissue sections were stained with uranyl acetate and lead citrate and examined with the aid of an electron microscope (JEM-1400 Plus, Japan). Electron microscopic studies were performed on five samples from each group.

Free fatty acid induced lipotoxicity assay

HepG2 cells $\left(2 \times 10^{4}\right)$ were seeded onto 96-well plate and incubated at $37^{\circ} \mathrm{C}, 5 \% \mathrm{CO} 2$ incubator. After $24 \mathrm{~h}$, media were removed and palmitic acid $(400 \mu \mathrm{M})$ was added to induce lipotoxicity. After $24 \mathrm{~h}$, palmitic acid was removed, and low and high doses of sericin were added to the cells. After $24 \mathrm{~h}$, cells were washed with phosphate-buffered saline, and CCK-8 solution $(100 \mu \mathrm{l} /$ well $)$ was added 
and cells were incubated for one hour. After one hour, the absorbance was measured at $450 \mathrm{~nm}$ using microplate reader.

\section{Mitochondria membrane potential assay}

For comparing mitochondria membrane potential, HepG2 cells $\left(1 \times 10^{5}\right)$ were seeded onto a 6-well plate and incubated for a day. After stabilization, JC-1 Solution (CS0390, Sigma-Aldrich) mixed with equal volume of complete media, was applied and incubated in $37^{\circ} \mathrm{C}, 5 \% \mathrm{CO} 2$ incubator for 20 mins. After aspiration of staining mixture, cells were washed with growth medium, covered with cover slip with mounting solution and observed under fluorescent microscope.

\section{Statistical analysis}

All data are expressed as the mean \pm standard error. The differences between groups were analyzed using the Kruskal-Wallis test and were considered statistically significant at $\mathrm{p}<0.05$.

\section{Results}

Sericin decreases liver weight and liver to bodyweight ratio

There was no difference in body weights between NASH (49.12 $\pm 1.85 \mathrm{~g})$ and $\mathrm{NASH}+\mathrm{S}$ groups $(47.56 \pm 1.63 \mathrm{~g} ; \mathrm{p}=0.6392)$ at the end of study (Figure 1B). Besides, there was also no difference in dietary intake between $\mathrm{NASH}$ and $\mathrm{NASH}+\mathrm{S}$ groups. Overall, liver weight was significantly lower in $\mathrm{NASH}+\mathrm{S}$ group $(2.05 \pm 0.39 \mathrm{~g})$ than NASH group $(2.61 \pm 0.36 \mathrm{~g} ; \mathrm{p}=0.0143)$. Liver to body weight ratio percentage was also significantly lower in NASH+S group $(4.30 \pm 0.69 \mathrm{~g})$ compare to NASH group $(5.26 \pm 0.62 \mathrm{~g} ; \mathrm{p}=0.0141)$.

Sericin improves hepatic enzymes and blood glucose levels

Sericin administration resulted in significantly decreased serum ALT (262.2 vs. $329.5 \mathrm{U} / \mathrm{L}, \mathrm{p}=0.0311$ ), AST (205.1 vs. $344.1 \mathrm{U} / \mathrm{L}, \mathrm{p}=$ 0.0423 ), and TG levels (43.8 vs. $62.6 \mathrm{mg} / \mathrm{dL}, \mathrm{p}=0.0321$ ), compared to the NASH group (Figure 1C). There were no significant differences in serum albumin and cholesterol levels between $\mathrm{NASH}+\mathrm{S}$ and NASH groups. OGTT revealed decreased blood glucose levels especially after 30 minutes $(\mathrm{p}=0.0199)$, in $\mathrm{NASH}+\mathrm{S}$ group. The area under the curve (AUC) of the plasma glucose concentration tended to be lower in $\mathrm{NASH}+\mathrm{S}$ group (Figure 1D).

A

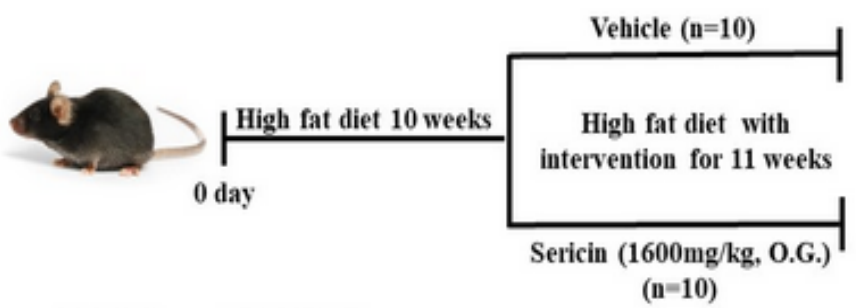

B

NASH NASH+S
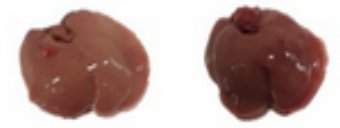

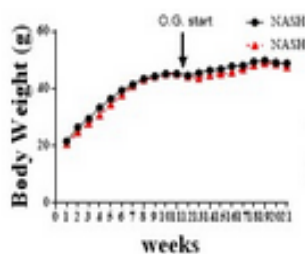

weeks

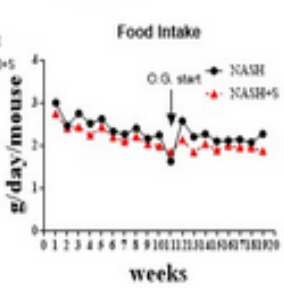

weeks
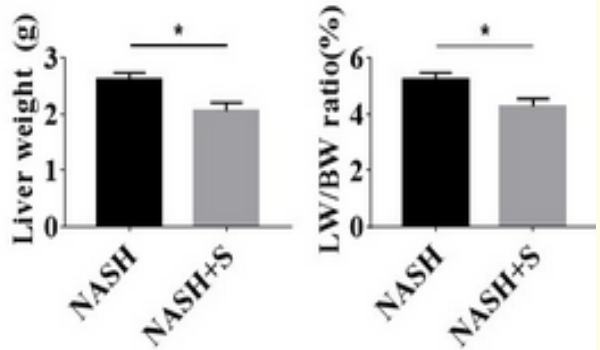

Citation: Dae Won Jun., et al. "Sericin Peptide Attenuates Non-Alcoholic Steatohepatitis Via Mitochondrial Function Modulation". Acta Scientific Nutritional Health 4.9 (2020): 42-52. 
C
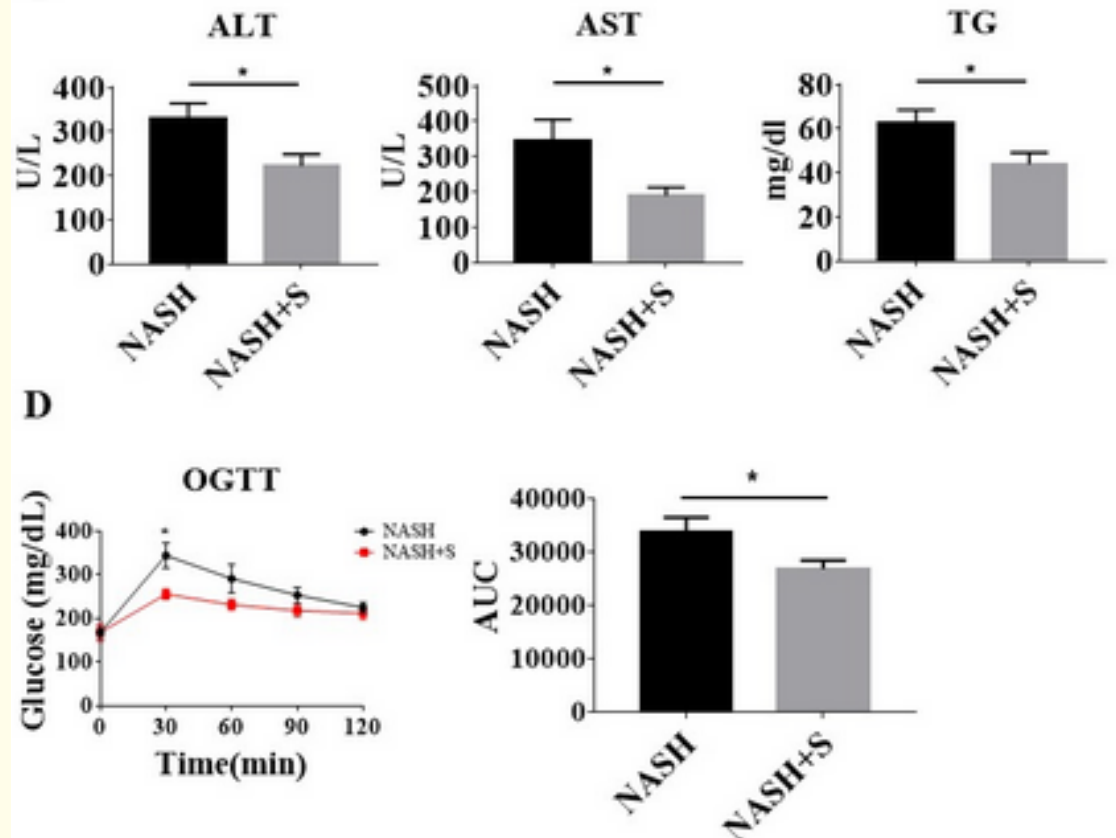

Figure 1: Study design, body weight, food intake, and liver to body weight ratio.

(1A) HF diet ( $60 \% \mathrm{kcal})$ was fed to mice for 21 weeks. From $11^{\text {th }}$ to $21^{\text {st }}$ week sericin and vehicle were administered to mice.

(1B) Body weight and food intake were measured weekly. At the end of experiment, liver weight and liver to body weight ratio was measured.

NASH: Non-Alcoholic Steatohepatitis; NASH+S: Sericin Treatment in NASH; LW/BW: liver weight to body weight.

(1C) NASH-sericin group showed a significantly decreased serum ALT (262.2 vs. $329.5 \mathrm{U} / \mathrm{L}, \mathrm{p}=0.0311$ ), AST (205.1 vs. $344.1 \mathrm{U} / \mathrm{L}$, $\mathrm{p}$ $=0.0423$ ), and TG levels (43.8 vs. $62.6 \mathrm{mg} / \mathrm{dL}, \mathrm{p}=0.0321$ ), compared to the NASH group.

(1D) OGTT revealed decreased blood glucose levels especially after 30 minutes ( $p=0.0199$ ), in NASH+S group. The area under the curve (AUC) of the plasma glucose concentration tended to be lower in NASH+S group.

NASH: non-alcoholic steatohepatitis; NASH+S: sericin treatment NASH; NAS: NAFLD activity score; ALT: Alanine Aminotransferase; AST: Aspartate Aminotransferase; TG: Triglycerides.

Sericin improves hepatic steatosis, NAS, and hepatic fibrosis

In $\mathrm{NASH}+\mathrm{S}$ group, the degree of hepatic steatosis was significantly improved compared to NASH group ( $\mathrm{p}=0.0002$ ) (Figure 2A-a, 2A-b and 2B-a). Although hepatocyte ballooning and lobular inflammation were also decreased in $\mathrm{NASH}+\mathrm{S}$ group compared to NASH group; however, the difference was not significant (Figure 2B-b, 2B-c). On H\&E stain, hepatocyte ballooning was noted more frequently in NASH group than in $\mathrm{NASH}+\mathrm{S}$ group (Figure 2A-c, 2Ad).
The overall NAS score was also significantly reduced $(\mathrm{p}=$ 0.0065 ) in NASH+S group (3.29) compared to NASH group (5.38) (Figure 2B-d). Moreover, overall fibrosis was much reduced in $\mathrm{NASH}+\mathrm{S}$ group than in NASH group (Figure 2A-e, 2A-f). Total score of fibrosis stage was significantly lower in $\mathrm{NASH}+\mathrm{S}$ group than NASH group ( $\mathrm{p}=0.0406$ ) (Figure 2B-e). Also, fibrosis percentage area was greater in NASH group (1.99) than in NASH+S group (1.29) ( $\mathrm{p}=0.0298)$ (Figure 2B-f). 
A

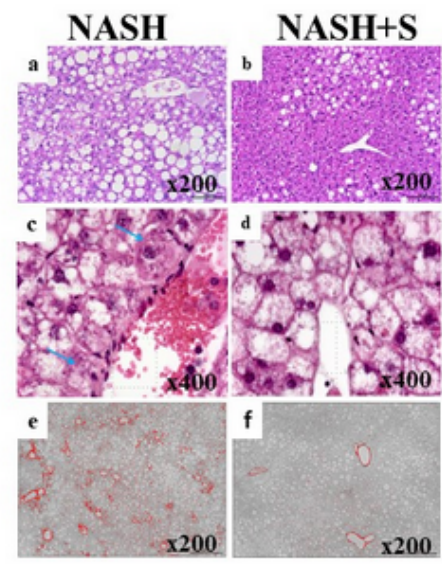

B

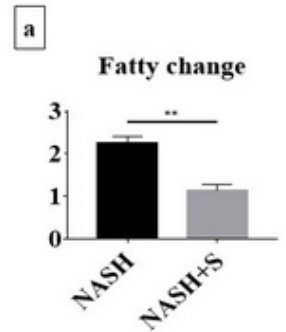

b

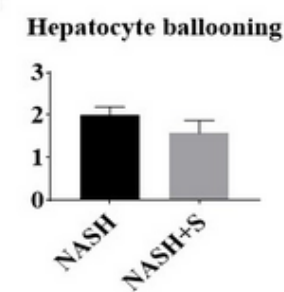

c

Lobular inflammation

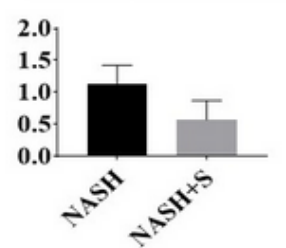

d

e
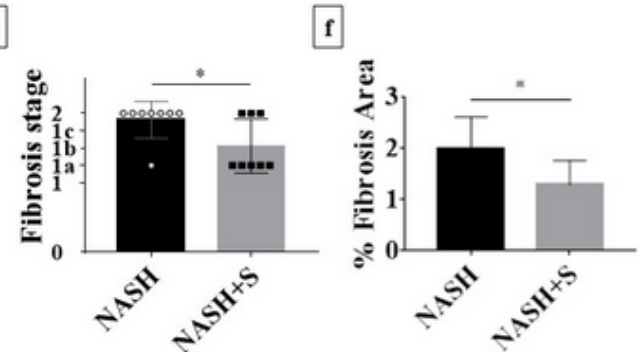

Figure 2: Histological and biochemical assessment of control and sericin groups.

(2A-a) Hepatic steatosis and lobular inflammation are noted in NASH group and (2A-b) steatosis was significantly reduced in NASH+S group (H\&E, X200).

(2A-c) Ballooning degeneration (arrows) of hepatocytes with Mallory Denk bodies are shown in zone3 near steatotic hepatocytes in NASH group, but (2A-d) not evident in NASH+S group (H\&E, X400).

(2A-e) Sirius-red staining highlights perivenular, perisinusoidal fibrosis of zone 3 as well as portal and periportal fibrosis in NASH group, in contrast to (2A-f) delicate strands of collagen deposition in the perivenular, perisinusoidal spaces in NASH-sericin group (X200).

(2B) H\&E stain stained liver tissue sections were evaluated for overall (2B-d) NAS score; (2B-a) degree of fatty change, (2B-b) hepatocytes ballooning and (2B-c) inflammation, and (2B-e, 2B-f) fibrosis. Fatty change, NAS score and fibrosis were significantly reduced in $\mathrm{NASH}+\mathrm{S}$ group than in NASH group. 


\section{Sericin decreases inflammation and de novo fat synthesis}

mRNA expressions of tumor necrosis factor alpha (TNF- $\alpha$ ), monocyte chemoattractant protein-1 (MCP-1) and interleukin-6 (IL-6), which are liver inflammation markers, were also significantly decreased in NASH+S group. mRNA expressions of hepatic de novo lipogenesis genes including sterol-regulatory-elementbinding protein-1c (SREBP1c), fatty acid synthase (FAS), and stearoyl-CoA desaturase-1 (SCD-1) were also significantly decreased in $\mathrm{NASH}+\mathrm{S}$ group than NASH group (Figure $3 \mathrm{~A}$ ).

\section{Sericin prevents abnormality of mitochondria}

In hepatocytes of NASH group, lipid droplets and abnormal mitochondria were frequently observed than in NASH+S group (Figure 3B-a). Abnormal mitochondria include megamitochondria, the presence of needle-like or electron dense inclusions, the loss of inner cristae, mitochondria swelling with matrix cavitation, and wall rupture (Figure 3B-b). In contrast, in $\mathrm{NASH}+\mathrm{S}$ group, the hepatocytes contained increased accumulation of glycogen particles and reduced numbers of lipid droplets and megamitochondria (Figure 3B-c). Moreover, mitochondria are relatively well preserved and often contained glycogen particles (Figure 3B-d).

Sericin attenuates FFA-induced cell death

Palmitate induced decreased HepG2 cells survival was improved with high dose of sericin $(\mathrm{p}<0.05)$ (Figure 4A). Mitochondrial membrane potential was measured using Z0-1. ZO-1 expression decreased in presence of palmitic acid. In contrast, sericin prevented mitochondrial depolarization (Figure 4B).

\section{Discussion and Conclusion}

In current study, we investigated the effects of sericin, a silkworm extract, on NASH. We observed that sericin peptide attenuated NASH via mitochondrial function modulation.

Okazaki., et al. reported that in high fat diet fed rats, sericin lowered phospholipids, free fatty acids, very-low-density lipoprotein

A
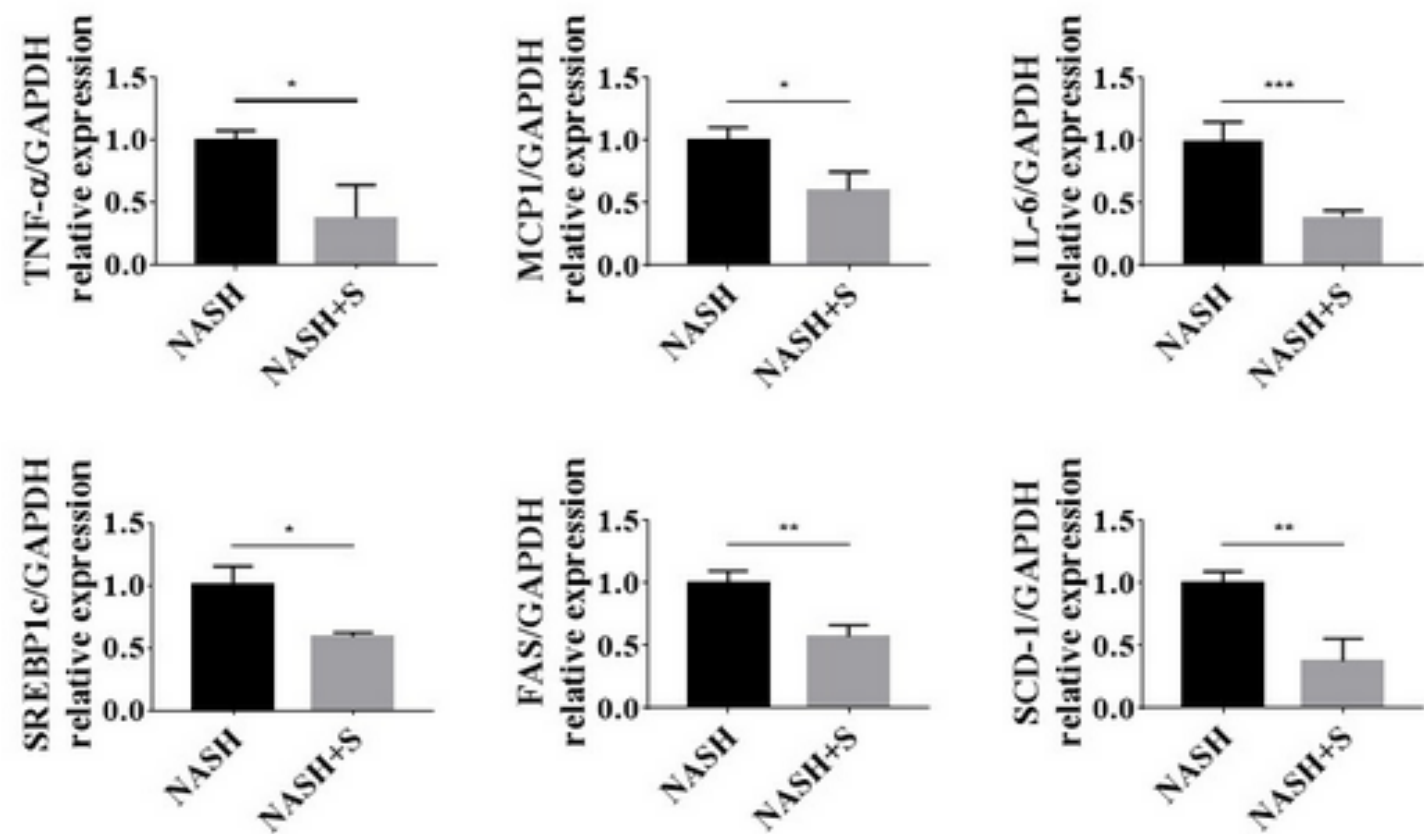
B

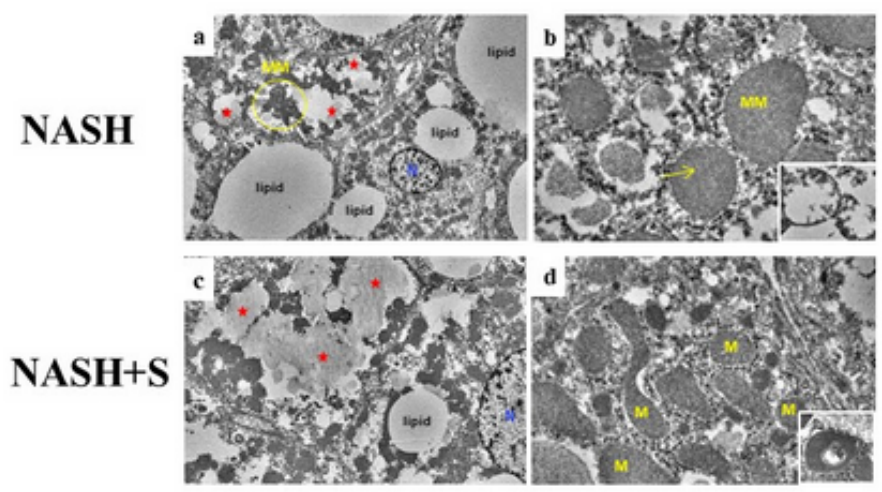

Figure 3: qPCR and electron microscopic findings in hepatocytes from NASH (upper panel) and NASH+S groups (lower panel).

(3A) mRNA expressions of liver inflammation markers (TNF- $\alpha$, MCP-1 and IL-6) and hepatic de novo lipogenesis genes including SREBP1c, FAS, and SCD-1 were significantly decreased in NASH+S group than NASH group.

(3B-a) In hepatocytes of NASH group, lipid droplets and abnormal mitochondria were frequently observed (x4000).

(3B-b) Abnormal mitochondria include megamitochondria(MM), the presence of needle-like (arrow) or electron dense inclusions, the loss of inner cristae (X40000), mitochondria swelling with matrix cavitation, and wall rupture (inset, X30000). In contrast, in $\mathrm{NASH}+\mathrm{S}$ group, (3B-c) increased accumulation of glycogen particles and reduced numbers of lipid droplets and megamitochondria were seen (X7000). (3B-d) Moreover, mitochondria were relatively well preserved (X20000) with often glycogen deposition (asterisk) (inset, X30000).

M: Mitochondria; MM: Megamitochondria; arrow: Needle Like Inclusion; asterisk: Glycogen Deposition ; N: Nucleus.

\section{A}

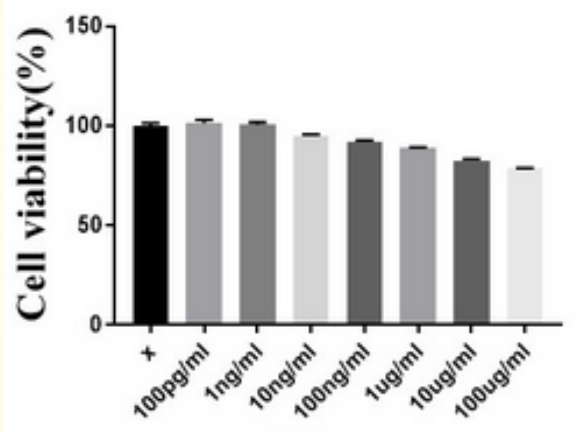

Sericin conc.
$\mathrm{PA}: 200 \mu \mathrm{M}$

Sericin low $(+): 100 \mathrm{pg} / \mathrm{mL}$

Sericin high $(++): 10 \mathrm{ng} / \mathrm{mL}$

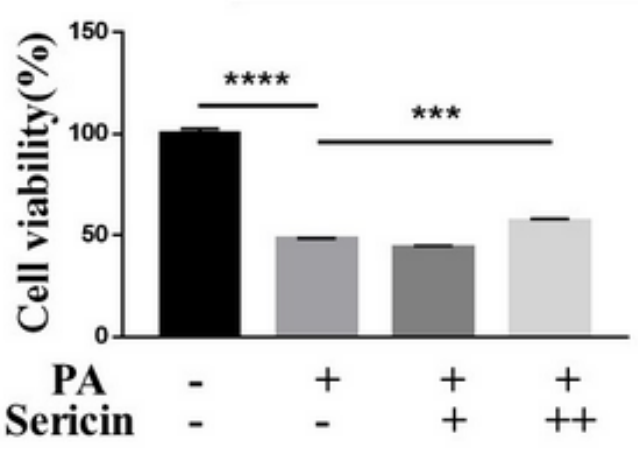




\section{B}
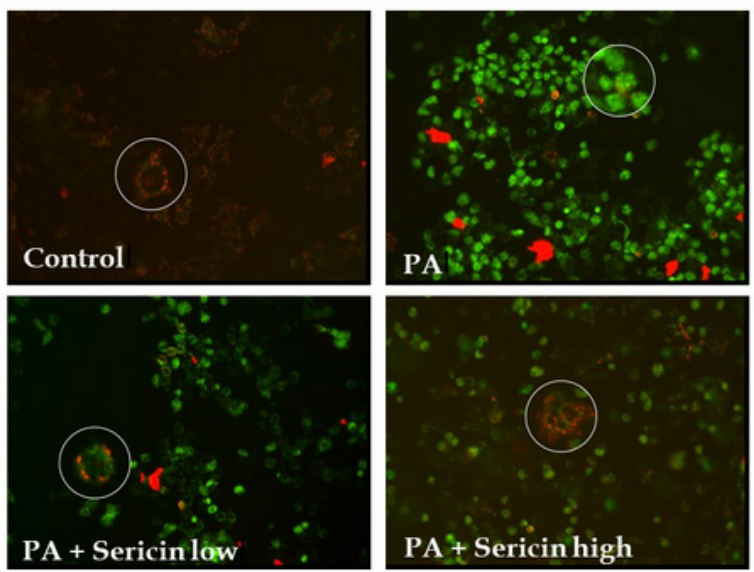

Figure 4: Free fatty acid induced lipotoxicity assay to evaluate HepG2 cells viability and confocal microscopic assessment of mitochondrial membrane potential.

(4A) No signs of toxicity were observed in HepG2 cells exposed to upto $100 \mu \mathrm{g} / \mathrm{mL}$ sericin. High dose sericin (10 ng/mL) and palmitic acid co-treated HepG2 cells had increased viability.

(4B) Mitochondrial membrane potential was assessed using ZO-1 staining. Viable mitochondria are seen as red dots in the cytoplasm. Sericin prevented mitochondrial depolarization.

PA concentration: $200 \mu \mathrm{M}$; Sericin low (+): 100pg/mL; Sericin high (++): $10 \mathrm{ng} / \mathrm{mL}$.

(VLDL) TG, VLDL cholesterol, low-density lipoprotein (LDL) cholesterol, and LDL phospholipid levels as well as blood TG and cholesterol levels [10]. In agreement with our results, they found no change in body weight between experimental and control groups. However, in our study, blood cholesterol levels were not significantly decreased in treatment group. Okazaki., et al. also showed that in NAFLD model, sericin inhibits hepatic lipogenesis and reduced serum free fatty acid levels. However, sericin administration also resulted in increased adiponectin levels in blood. As adiponectin has protective effects on hyperlipidemia, fatty liver, and glucose intolerance; in rats sericin treatment decreased blood glucose and insulin levels after intraperitoneal injection of glucose. In our study, sericin-treated mice also had significantly decreased blood glucose levels and area under the curve (AUC) 30 minutes after glucose oral gavage, suggesting that sericin lowers insulin resistance.

Several studies reported that sericin can regulate hepatic fat, blood cholesterol, and oxidative stress by regulating mitochon- drial function. Sericin lowered cholesterol by several mechanisms including anti-oxidative effects and reduced intestinal cholesterol absorption. Ampawong., et al. have shown that sericin has antioxidant properties and it reduced fat accumulation in hepatocytes, blood cholesterol, and dysmorphic mitochondria in rats subjected to high-cholesterol diet and streptozotocin treatment [8]. Sericin induced improvements were thought to be mediated by its antioxidant properties [8]. Lapphanichayakool., et al. also explained that sericin administration affects cholesterol solubility of bile salts, reduces cholesterol absorption in intestine and, consequently, blood cholesterol levels [11]. Deori., et al. demonstrated that sericin reduces cholesterol reabsorption by increasing bowel movements. Another study also demonstrated that sericin decreased thiobarbituric acid reactive substances, the oxidative stress marker, levels and increased endogenous antioxidant levels [12]. Sericin induced protective mechanism preserves mitochondrial structure and lowers cholesterol levels which alleviates hepatic steatosis and improves liver function. Our data also supported observations from 
previous studies. Electron microscopic findings showed restoration of abnormal mitochondria in livers of sericin-treated mice. Besides, sericin also prevented mitochondrial depolarization after palmitic acid treatment. Moreover, hepatic glycogen accumulation was increased in $\mathrm{NASH}+\mathrm{S}$ group compared to NASH group.

The relationship between insulin resistance and mitochondrial function has been continuously studied $[2,13]$. Insulin resistance is associated with mitochondrial dysfunction via decreased mitochondrial activity, decreased fatty acid oxidation, and increased diacylglycerol levels in patients with type 2 diabetes [14]. Insulin resistance is known to be closely associated with mitochondrial abnormalities. Sericin induced improved insulin resistance is thought to be mediated by mitochondrial function preservation. In our study, sericin inhibited mitochondrial dysfunction and structural changes. In addition, sercin also significantly decreased inflammatory cytokines, TNF- $\alpha$, MCP-1, and IL-6, suggesting that sericin prevents inflammation and NASH progression.

mRNA expressions of hepatic de novo lipogenesis genes such as SREBP1c, FAS, and SCD-1 were also significantly decreased in $\mathrm{NASH}+\mathrm{S}$ group than in NASH group. Fibrosis stage was lowered and quantified fibrosis area was decreased in NASH+S group.

In conclusion, in NASH animal model, sericin reduced intrahepatic fat, mitochondrial injury, inflammation and fibrosis. Sericin induced improvement in NASH was due to decreased mitochondrial abnormality. There is a need for further evaluation regarding the potential of sericin in modulating mitochondrial signaling and function and its therapeutic utility in NASH.

\section{Ethics Approval and Consent to Participate}

The experimental protocol was approved by the Institutional Animal Care and Use Committee of Hanyang University (HY-IACUC-18-0042).

\section{Consent for Publication}

I am the sole author of the manuscript. I am one author signing on behalf of all co-authors of the manuscript.

\section{Availability of Data and Material}

N/A.

\section{Competing Interests}

Hyukjin Kwon and Jaekyu Noh are full-time employees at NI\&Pharm INC.

\section{Funding}

This research was supported by grants from the Ministry of Food and Drug Safety in 2020 (20183MFDS525), and NI\&amp;Pharm INC.

\section{Authors' Contributions}

Dae Won Jun contributed to the study design; Na Ryung Choi wrote the manuscript; Hyeon Tae Kang did the molecular work and data management; Yeon-Lim Suh interpreted the histologic findings; and Sang Bong Ahn supervised the study.

\section{Acknowledgements}

This study was supported by grants from the Ministry of Food and Drug Safety (20183MFDS525) and by NI\&amp;Pharm INC. The sponsor, NI\&amp; Pharm INC, participated in the study's design but did not have any role in the implementation, data collection, analysis, or interpretation of the manuscript.

\section{Bibliography}

1. Li J., et al. "Prevalence, Incidence, and Outcome of Non-Alcoholic Fatty Liver Disease in Asia, 1999-2019: A Systematic Review and Meta-Analysis". Lancet Gastroenterology and Hepatology 4.5 (2019): 389-398.

2. Lim CY., et al. "Effects of Carnitine on Peripheral Blood Mitochondrial DNA Copy Number and Liver Function in Non-Alcoholic Fatty Liver Disease". Korean Journal of Gastroenterology 55.6 (2010): 384-9.

3. Oh H., et al. "Non-Alcoholic Fatty Liver Diseases: Update on the Challenge of Diagnosis and Treatment". Clinical and Molecular Hepatology 22.3 (2016): 327-335.

4. Simoes ICM., et al. "Mitochondria in Non-Alcoholic Fatty Liver Disease". The International Journal of Biochemistry and Cell Biology 95 (2018): 93-99.

5. Nassir F and JA Ibdah. "Role of Mitochondria in Nonalcoholic Fatty Liver Disease". International Journal of Molecular Sciences 15.5 (2014): 8713-8742.

6. Kundu Subhas C., et al. "Natural Protective Glue Protein, Sericin Bioengineered by Silkworms: Potential for Biomedical and Biotechnological Applications". JPPS Progress in Polymer Science 33.10 (2008): 998-1012.

7. Padol Amol., et al. "Natural Biomaterial Silk and Silk Proteins: Applications in Tissue Repair". International Journal of Materials and Biomaterials Applications 2 (2012): 19-24. 
8. Ampawong S., et al. "Sericin Ameliorated Dysmorphic Mitochondria in High-Cholesterol Diet/Streptozotocin Rat by Antioxidative Property". Experimental Biology and Medicine (Maywood) 242.4 (2017): 411-421.

9. Kleiner DE., et al. "Design and Validation of a Histological Scoring System for Nonalcoholic Fatty Liver Disease". Hepatology 41.6 (2005): 1313-1321.

10. Okazaki Y., et al. "Consumption of Sericin Reduces Serum Lipids, Ameliorates Glucose Tolerance and Elevates Serum Adiponectin in Rats Fed a High-Fat Diet". Bioscience, Biotechnology, and Biochemistry 74.8 (2010): 1534-1538.

11. Lapphanichayakool P., et al. "Hypocholesterolemic Effect of Sericin-Derived Oligopeptides in High-Cholesterol Fed Rats". Journal of Natural Medicines 71.1 (2017): 208-215.

12. Deori M., et al. "Antioxidant Effect of Sericin in Brain and Peripheral Tissues of Oxidative Stress Induced Hypercholesterolemic Rats". Frontiers in Pharmacology 7 (2016): 319.

13. Jun DW., et al. "Prevention of Free Fatty Acid-Induced Hepatic Lipotoxicity by Carnitine Via Reversal of Mitochondrial Dysfunction". Liver International 31.9 (2011): 1315-24.

14. Gonzalez-Franquesa A and ME Patti. "Insulin Resistance and Mitochondrial Dysfunction". Advances in Experimental Medicine and Biology 982 (2017): 465-520.

\section{Assets from publication with us}

- Prompt Acknowledgement after receiving the article

- Thorough Double blinded peer review

- Rapid Publication

- Issue of Publication Certificate

- High visibility of your Published work

Website: www.actascientific.com/

Submit Article: www.actascientific.com/submission.php

Email us: editor@actascientific.com

Contact us: +919182824667

Citation: Dae Won Jun., et al. "Sericin Peptide Attenuates Non-Alcoholic Steatohepatitis Via Mitochondrial Function Modulation". Acta Scientific Nutritional Health 4.9 (2020): 42-52. 